\title{
The Three Hundred Project: The Influence of Environment on Simulated Galaxy Properties
}

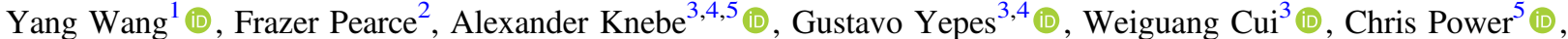 \\ Alexander Arth ${ }^{6,7}$, Stefan Gottlöber ${ }^{8}$, Marco De Petris ${ }^{9}$, Shaun Brown ${ }^{2}$, and Longlong Feng ${ }^{1}$ \\ ${ }^{1}$ School of Physics and Astronomy, Sun Yat-sen University, 519082, Zhuhai, People's Republic of China; wangyang23@mail.sysu.edu.cn \\ ${ }^{2}$ School of Physics \& Astronomy, University of Nottingham, Nottingham NG7 2RD, UK \\ ${ }^{3}$ Departamento de Física Teórica, Módulo 8, Facultad de Ciencias, Universidad Autónoma de Madrid, E-28049 Madrid, Spain \\ ${ }^{4}$ Centro de Investigación Avanzada en Física Fundamental (CIAFF), Universidad Autónoma de Madrid, E-28049 Madrid, Spain \\ ${ }^{5}$ International Centre for Radio Astronomy Research, The University of Western Australia, 35 Stirling Highway, Crawley, Western Australia 6009, Australia \\ ${ }^{6}$ University Observatory Munich, Scheinerstraße 1, D-81679 Munich, Germany \\ ${ }^{7}$ Max-Planck-Institute for Extraterrestrial Physics, Giessenbachstraße 1, D-85748 Garching, Germany \\ ${ }^{8}$ Leibniz-Institut für Astrophysik, D-14482 Potsdam, Germany \\ ${ }^{9}$ Department of Physics, Sapienza Università di Roma, p.le Aldo Moro 5, I-00185 Rome, Italy \\ Received 2018 July 20; revised 2018 September 23; accepted 2018 September 28; published 2018 November 30
}

\begin{abstract}
The relationship between galaxy properties and environment is a widely discussed topic within astrophysics. Here, we use galaxy samples from hydrodynamical resimulations to examine this relationship. We use the overdensity $\left(\delta_{1}\right)$ within a $1 h^{-1} \mathrm{Mpc}$ sphere around a galaxy to evaluate its environment. Then, the relations between galaxy properties, such as specific star formation rate (sSFR), fraction of star-forming galaxies, $g-r$ color, and $\delta_{1}$ are examined within three galactic samples formed from galaxies within large clusters, those in the vicinity of large clusters, and those in the field. We find tight environmental correlations for these galaxy properties. In brief, galaxies in denser environments tend to be redder and are more likely to be quenched; this is consistent with observations. We find that although the sSFR decreases with $\delta_{1}$, this is mainly because galaxies with higher stellar mass reside in an environment with higher overdensity. At fixed overdensity, a galaxy's color is also independent of whether it lives within a cluster or within the field, but the relative fractions of the two samples varies dramatically with overdensity, and this drives an apparent evolution.
\end{abstract}

Key words: galaxies: clusters: general - galaxies: evolution - methods: numerical

\section{Introduction}

An important issue for the study of galaxy formation is the relationship between a galaxy's properties and the environment it inhabits. This issue was perhaps first studied in the pioneering work of Oemler (1974) and Dressler (1980). Their work identified and quantified a morphology-density relationship, showing that spiral galaxies prefer to reside in lowerdensity environments than elliptical and S0 galaxies. Later work investigated this relationship in more detail, and explored star formation, color, and morphology as additional discriminants. Many of these studies support a strong relationship between galactic properties and environmental density (e.g., Kauffmann et al. 2004; Tanaka et al. 2004; Elbaz et al. 2007; Peng et al. 2010; De Lucia et al. 2012a; Darvish et al. 2014). Such studies indicate that galaxies that are located in dense environments tend to be red, elliptical, and have lower star formation rates than their low-density counterparts (Lai et al. 2016).

The observed correlation between a galaxy's properties and the environment it lives in is thought to be due to interactions with their surroundings. Many physical processes will lead to the observed property-density relations, such as dynamical friction (Chandrasekhar 1943; Contini et al. 2012; De Lucia et al. 2012b), ram-pressure stripping (Gunn \& Gott 1972; Quilis et al. 2000; McCarthy et al. 2008), highspeed galaxy encounters (galaxy harassment; Moore et al. 1996), and galaxy-galaxy mergers (Mihos \& Hernquist 1994). However, due to the complexity of modeling the environmentally driven processes and the limitations of the observations, the relative significance of each possible environmental effect is still unclear. Selection effects and various approaches to characterizing the observed galactic density field introduce bias into the measured propertyenvironment relationship. On the other hand, any environmental effect is a combination of many physical processes, and each galaxy will experience various environments during its lifetime (see De Lucia et al. 2012b), which makes any theoretical calculation extremely complex. Thus, this is an interesting multifaceted problem from both a simulation and observational standpoint.

Several groups have investigated the influence of a galaxy's environment using semi-analytic models. For example, De Lucia et al. (2012b) studied the environmental history of group and cluster galaxies. They found that the stellar mass and star formation rate (SFR) could be related to the environment a galaxy inhabited before it was accreted into its final dark-matter halo. They quantified that such "preprocessing" had an effect on $27 \%-44 \%$ of their group/cluster galaxies, with the percentage varying for galaxies with different stellar mass. It has also been suggested that the observed difference in the fraction of passive galaxies in different clusters, as well as some other properties of galaxies, (e.g., Weinmann et al. 2011; De Lucia et al. 2012a), could be related to halo-to-halo scatter in the way that the final halo was assembled (essentially a wide variation in the range of possible halo merger trees).

However, semi-analytic models do not resolve all the complex physical processes involved in galaxy formation, as they generally prescribe some of the relations between a galaxy 
and its host halo. These relations may combine the effects of many physical processes, and decomposing them into analytical schema can prove difficult. On the other hand, exploring the environment-galaxy relationship with semi-analytic models can be undertaken even if the halo-galaxy relationship is manually determined, so long as care is taken not to simply recover an input relationship. To go beyond this, cosmological hydrodynamical simulations are commonly used. Hydrodynamical simulations, which include baryonic processes operating under a self-consistent gravitational framework, directly encode many of the physical processes that may impinge upon galaxy formation and evolution. Until recently, there have not been many studies investigating the galaxy property-environment relationship using hydrodynamical simulations. Gabor \& Davé (2012, 2015) reproduced the environmental dependence of galaxy color and quenching within a hydrodynamical simulation, finding a larger fraction of red galaxies within denser environments. Their results indicate that satellite galaxies are affected by environmental quenching, while the quenching process operating for central galaxies is largely driven by their own stellar mass. Later work (Vogelsberger et al. 2014) investigated the same processes as Gabor \& Davé (2012, 2015) within the larger ILLUSTRIS simulation. Both these studies agreed with the observational results from SDSS and zCosmos surveys (Peng et al. 2010) quite well. Later, Rafieferantsoa et al. (2015) investigated the relationship between H I content and local overdensity in a large simulation. This work indicated that the median $\mathrm{HI}$ and specific star formation rate (sSFR) drops in denser environments, whether quenched galaxies are taken into account. This result is also consistent with observational studies (Fabello et al. 2012). M. Lotz et al. (2018, in preparation), utilizing the MAGNETICUM simulation, found that star-forming galaxies are quenched during their first passage. Furthermore, they find that quenching is impeded at high stellar mass, suggesting that the mass of such galaxies effectively shields them from ram-pressure stripping. While hydrodynamical simulations, such as those mentioned above, are considered a vital tool in aiding and interpreting astronomical observations of galaxy clusters (Borgani \& Kravtsov 2011), the detailed effect of baryonic physics remains unclear, especially on small scales (e.g., see Cui et al. 2016; Cui \& Zhang 2017, for a review of baryonic effects). Furthermore, the use of different simulation codes and techniques and the inclusion (or not) of different physical processes adds additional uncertainty. The nIFTy galaxy cluster comparison project (Arthur et al. 2016; Cui et al. 2016; Elahi et al. 2016; Sembolini et al. 2016a, 2016b) compared a dozen common simulation codes by simulating one identical galaxy cluster to investigate their differences. They found that although the dark-matter-only runs gave quite good agreement between different simulations (Sembolini et al. 2016a); the hydrodynamical runs showed a large discrepancy, especially when different baryonic models are included (Cui et al. 2016). Because one of the main issues with the nIFTy comparison was the potential for cluster-to-cluster scatter, the THREE HUNDRED PROJECT aimed to increase the galaxy cluster sample to 324 large clusters. It focuses on the statistical results for both hydrodynamical simulations and semi-analytical galaxy formation models of the clusters (Cui et al. 2018). As one work in this project, we focus on quantifying any environmental effect on the galaxies within our hydrodynamical simulations. Two codes, Gadget-X and GADGET-MUSIC, are employed for running the resimulations. As well as the 324 cluster resimulations, we also simulate four large field regions specifically targeted so as not to include any significant cluster. This will allow us to explore the possible influence from largescale structures. We intend to study the relationship between galaxy properties and their large-scale environment.

The remainder of this paper is organized as follows. In Section 2 we describe the simulations we have used and the definition of the extracted galaxy properties and environmental density. In Section 3 we present the results of our analysis: subsection 3.1 discusses the relation between SFR and environmental overdensity, and subsection 3.2 shows the environmental dependence of a galaxy's color and magnitude. Finally, we draw conclusions in Section 4.

\section{Simulation Data}

This paper utilizes the simulation data set provided by the THREE HUNDRED PROJECT. This consists of 324 re-simulated clusters and four field regions extracted from within the MultiDark Planck simulation, MDPL2, (Klypin et al. 2016). The MDPL2 simulation has cosmological parameters of $\Omega_{M}=0.307, \quad \Omega_{B}=0.048, \quad \Omega_{\Lambda}=0.693, \quad h=0.678, \quad \sigma_{8}=$ 0.823 . All the clusters and fields have been simulated using the full-physics hydrodynamical codes GADGET-X and GADGET-MUSIC, which are updated versions of GADGET2 (Springel 2005). In the re-simulation region, the mass of a dark-matter particle is $12.7 \times 10^{8} h^{-1} M_{\odot}$ and the mass of a gas particle is $2.36 \times 10^{8} h^{-1} M_{\odot}$. The mass of a star particle varies from $3.60 \times 10^{7} h^{-1} M_{\odot}$ to $1.65 \times 10^{8} h^{-1} M_{\odot}$ with $99 \%$ of the star particles being less massive than $4.60 \times 10^{7} h^{-1} M_{\odot}$. Each cluster re-simulation consists of a spherical region of radius $15 h^{-1} \mathrm{Mpc}$ at $z=0$ centered on one of the 324 largest objects from within the host MDPL2 box, which is $1 h^{-1} \mathrm{Gpc}$ on a side. The host halos of our galaxies range in mass from $2.54 \times 10^{10} h^{-1} M_{\odot}$ to $2.63 \times 10^{15} h^{-1} M_{\odot}$. The largest halo within each of the 324 cluster resimulations varies from $8.15 \times 10^{14} h^{-1} M_{\odot}$ to $2.63 \times 10^{15} h^{-1} M_{\odot}$. The field regions are low-density volumes of radius $43 h^{-1} \mathrm{Mpc}$ selected so as not to include any halos larger than $4 \times 10^{13} h^{-1} M_{\odot}$. Although not technically selected to be voids they are all underdense relative to the cosmic mean. Detailed descriptions of the 324 clusters and the simulation codes can be found in Cui et al. (2018). We also refer interested readers to Beck et al. (2016) for details of GADGET-X and Sembolini et al. (2013) for details of GADGETMUSIC. In summary, GADGET-X uses an improved SPH scheme (Beck et al. 2016), while GADGET-MUSIC uses a classic SPH scheme; GADGET-X includes a full-physics baryon model including AGN feedback, while GADGET-MUSIC does not take massive black hole growth or AGN feedback into account; in addition the models for stellar feedback are different. In this paper, we concentrate on the results of GADGET-X because, as we show below, this approach better reproduces the observed distribution of galaxy mass as a function of star formation rate.

To define a galaxy, we first use AHF (Knollmann \& Knebe 2009) to find halos and sub-halos within the simulations. Then a group of star and any associated gas particles inside a subhalo is defined as a galaxy. For central galaxies which do not belong to any sub-halos, we follow Furlong et al. (2015) by defining the group of all star and gas particles within $30 h^{-1} \mathrm{kpc}$ from the halo center as the central galaxy. While in principle a galaxy could contain as little as one star particle 
Table 1

Number of Galaxies at $z=0$ with Stellar Mass above $10^{9.5} h^{-1} M_{\odot}$ within Each Sample Class as Indicated for GADGET-X (Left Column) and GADGET-MUSIC (Right Column)

\begin{tabular}{lcc}
\hline \hline & GADGET-X & GADGET-MUSIC \\
\hline Cluster & 96470 & 133105 \\
Vicinity & 111662 & 186321 \\
Field & 7365 & 13724 \\
\hline
\end{tabular}

such poorly resolved galaxies will bring large uncertainties to any measurement of their properties. Thus, we constrain our galaxy samples by imposing a lower mass limit of $10^{9.5} M_{\odot} / h$, which roughly corresponds to 80 stars. We also excluded any galaxies whose host halo was contaminated by boundary particles. Boundary particles are low resolution particles used to surround the re-simulated region to preserve the influence of large-scale structure from the entire MDPL2 volume. As we need to calculate each galaxy's environment, we further abandon any galaxies lying too close to the boundary to avoid sampling any regions not populated by galaxies or contaminated with boundary particles. In another words, we only selected galaxies within $10 h^{-1} \mathrm{Mpc}$ of the center of cluster resimulations. For field resimulations, the corresponding distance is $38 h^{-1} \mathrm{Mpc}$.

The largest galaxy in the cluster resimulations has a stellar mass (including its associated intracluster light (ICL)) of $10^{13} h^{-1} M_{\odot}$. In the field regions this value is only $\sim 10^{11} h^{-1} M_{\odot}$. The number of galaxies in each sample group, above the indicated stellar mass, are represented in Table 1. The number of galaxies above a fixed stellar mass within the GADGETMUSIC simulations is almost twice that of GADGET-X. This is because, in the absence of AGN feedback, star formation within GADGET-MUSIC is more efficient. Essentially, as we see below, the galaxies within the GADGET-MUSIC models are more massive than their counterpart in the GADGET-X models.

Galaxies from our simulations are split into three categories based on their physical location. Galaxies located within twice $R_{200}$ of the cluster center are regarded as "cluster galaxies." Galaxies outside this radius in the cluster resimulations are regarded as "vicinity galaxies." Galaxies in the field resimulations contribute to the "field galaxy" sample. The re-simulation volume for consideration in the following text is always set to be a co-moving sphere with a radius of $10 h^{-1} \mathrm{Mpc}$ for the cluster resimulations or $38 h^{-1} \mathrm{Mpc}$ for the field resimulations. The center of these outer spheres is taken to be the center of the re-simulation region at $z=0$ and fixed for all time. The cluster center is found by AHF and moves with time. Hence the volume extracted as the "cluster region" moves in both location and size as the simulation evolves, although it remains well within the outer boundary at all times. The cluster "vicinity" region is all the volume that is outside the cluster region boundary and within the outer boundary.

To give a visual impression of our resimulations, images of the largest cluster and one of the field resimulations at $z=0$ are shown in Figure 1. Each circle represents a galaxy (with mass larger than $10^{9.5} h^{-1} M_{\odot}$ ) with the size of the circle proportional to the galactic stellar mass. The color represents the overdensity $\delta_{1}$ of its local environment, in this case within $1 h^{-1} \mathrm{Mpc}$ (see detailed definition of $\delta_{1}$ in Section 2.2). The bottom panels give an overview of the full re-simulated region. The top panels show a zoom into a $10(\mathrm{Mpc} / h)^{3}$ cube centered on the most massive galaxy. The blue dashed circle indicates the volume within which we could recover an accurate environmental overdensity for each galaxy. Galaxies outside the circle are excluded from our analysis. The radius of the blue circle is $10 h^{-1} \mathrm{Mpc}$ for cluster resimulations and $38 h^{-1} \mathrm{Mpc}$ for field resimulations. The two black circles indicate $R_{200}$ (solid) and $2 \mathrm{R}_{200}$ (dashed) of the most massive halo in each simulation. Their position and size will change through time. This figure also illustrates how our three galaxy classes are defined: in cluster resimulations, galaxies within the black dashed circles are classified as "cluster galaxies," and galaxies between the black dashed circle and the blue dash-dotted circle are classified as "vicinity galaxies." In field resimulations, all galaxies within the blue circle form the "field galaxies" sample. Note that the sizes of all the regions are given in co-moving units.

\subsection{Measurement of Galaxy Properties}

We study the environment's effect on a galaxy's SFR, luminosity (in the $r$ and $g$ bands), and color $\left(M_{g}-M_{r}\right)$. How we define and calculate each of these properties is given below:

1. SFR and SSFR: We define the SFR of a galaxy as the sum of the SFRs of all gas particles belonging to it. In the simulation, each gas particle has its own star formation rate which is determined by the star formation model within the simulation (Springel et al. 2005). At each step in the simulation, some fraction of the mass of a gas particle is converted into a new star particle according to its SFR. The sSFR(specific star formation rate) is defined as usual, $\mathrm{sSFR}=\mathrm{SFR} / M_{*}$.

2. LUMINOSITY and MAGNITUDE: The luminosity in any defined spectral band is calculated by applying the stellar population synthesis code STARDUST (see Devriendt et al. 1999, and references therein for more details). This code computes the spectral energy distribution from the far-UV to the radio. The stellar contribution to the total flux is calculated assuming a Kennicutt initial mass function (Kennicutt 1998). Absolute MAGNITUDES are readily calculated from the luminosity. Note dust obscuration is not taken into account. The THREE HUNDRED PROJECT gives the luminosities and magnitudes of galaxies in several bands. For this work we only use those derived from imposing Sloan Digital Sky Survey(SDSS) $g$ band and $r$ band filters.

\subsection{Measurement of a Galaxy's Environment}

Observationally, there are three methods commonly used to characterize a galaxy's environment:

1. Count the number of neighboring galaxies within a projected ring around each galaxy (e.g., Wilman et al. 2010).

2. Count the number of neighboring galaxies within a fixed volume around each galaxy (e.g., Kauffmann et al. 2004; Gallazzi et al. 2009; Grützbauch et al. 2011).

3 . Find the projected distance $r$ to the $n$th nearest neighbor galaxy, with $n$ in the range 3-10. Then, the projected surface density is calculated via $\Sigma_{n}=n / \pi r^{2}$ (e.g., Dressler 1980; Hashimoto et al. 1998; Tanaka et al. 2004; Capak et al. 2007). 


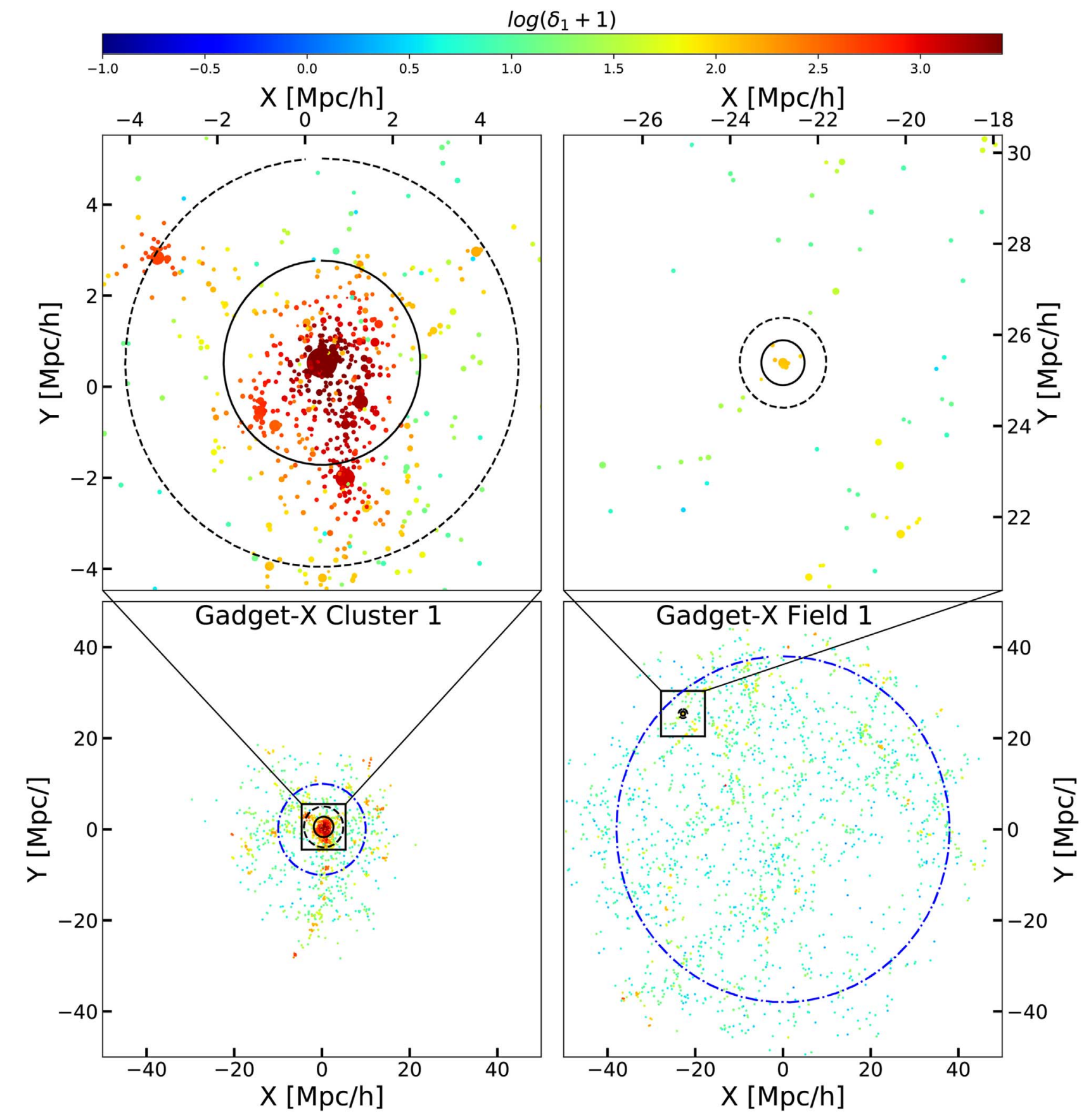

$\log \left(\delta_{1}+1\right)$

Figure 1. Distribution of galaxies within Cluster 1 and Field 1 at $z=0$, taken from the GADGET-X simulation. Each circle represents a galaxy with the circle's size proportional to its stellar mass. The environmental overdensity within $1 h^{-1} \mathrm{Mpc}\left(\delta_{1}\right)$ is indicated by the galaxy color. Only galaxies with stellar mass above $10^{9.5} h^{-1} \mathrm{Mpc}$ are shown. The bottom panels show an overview of the full region being re-simulated and to aid comparison have the same linear size. The top panels show a zoom into $10 h^{-1} \mathrm{Mpc}$ sided cubes centered on the most massive galaxy. The solid and dashed black circles indicate $R_{200}$ and twice $R_{200}$, the radius and double the radius of the most massive dark-matter halo in each re-simulation. In both cases, galaxies outside the blue dashed circle are excluded from the sample, as described in the text.

Further details and a comparison between various environmental estimators are given in Muldrew et al. (2012). As the above methods show, observationally the traditional parameter defining the environmental density is the local galaxy number density. However, as galaxies form a biased tracer of the density field (Mo \& White 1996), we might expect to find a more intrinsic environmental dependence if we utilise the underlying dark-matter density field. From observations, this underlying matter density field could be reconstructed from combining the galaxy mass density with a bias factor $b$. Galaxy mass density comes from weighting each galaxy with their mass in the formula of galaxy number density. However, the bias can be complicated depending on the scale, redshift, and type of galaxies included (Kovač et al. 2010). On the other hand, we can directly measure the actual density (smoothed on some scale) within a simulation.

In this work, we define $\delta_{1}$, the local overdensity of all matter (including stars, gas, and dark matter) compared with the mean density of the universe, within a sphere of radius $1 h^{-1} \mathrm{Mpc}$ centered on the galaxy to quantify the local environment. 


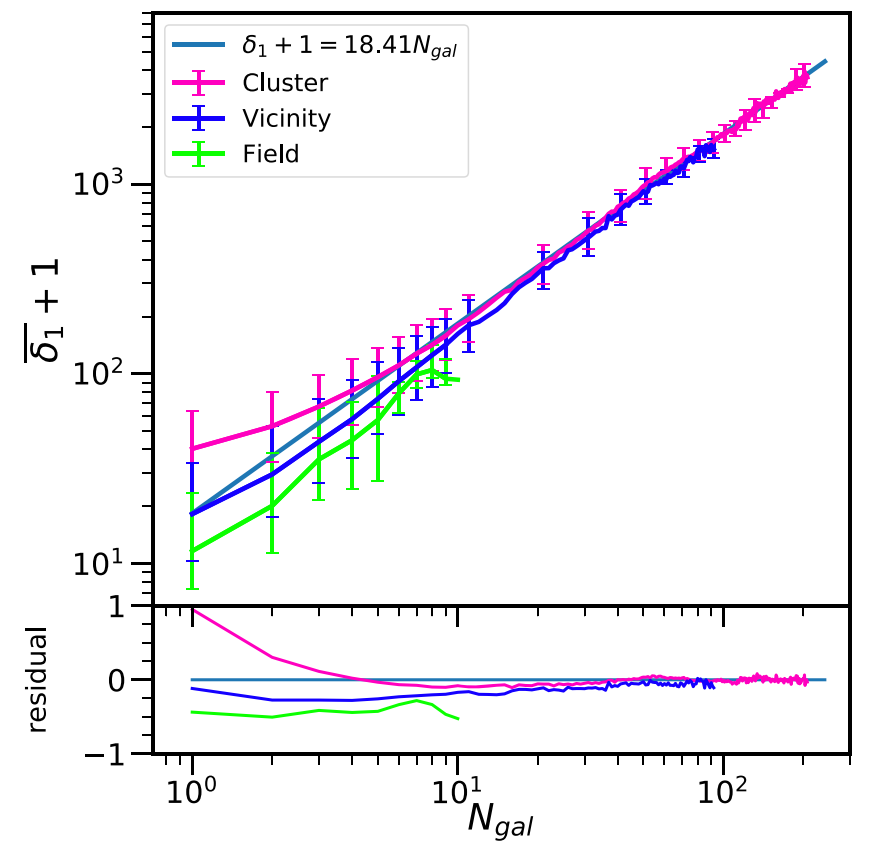

Figure 2. Median matter overdensity $\bar{\delta}$ as a function of galaxy number $N_{\text {gal }}$ within $1 h^{-1} \mathrm{Mpc}$ (top panel). The error bars show the 16th and 84th percentiles. The cyan line without error bars is a linear fit of $\delta$ directly proportional to $N_{\text {gal }}$ scaled to the cluster galaxy sample. In the lower panel, the residuals from the linear fit are shown. The residual is calculated as $\left(\delta_{1}-\delta_{f i t}\right) /\left(\delta_{f i t}+1\right)$.

$1 h^{-1} \mathrm{Mpc}$ is a characteristic radius of dark-matter halos with mass around $10^{13}-10^{14} h^{-1} M_{\odot}$. It is also a commonly used distance in the second method above for defining the environmental galaxy number density. This is the region that is thought to be tightly connected with a galaxy (Kauffmann et al. 2004). We have also measured the environment using radii of $2 h^{-1} \mathrm{Mpc}$ and $3 h^{-1} \mathrm{Mpc}$ to form $\delta_{2}$ and $\delta_{3}$. Such distances are widely used as the smoothing scale when calculating structural features such as nodes, filaments, sheets, and voids (e.g., Hahn et al. 2007; Zhang et al. 2009). We found that $\delta_{1}$ is linearly related to $\delta_{2}$ and $\delta_{3}$. The dependence of a galaxy's properties on $\delta_{2}$ and $\delta_{3}$ has almost the same shape as those found for $\delta_{1}$. Thus, in what follows, we only show the results for $\delta_{1}$.

To probe the relationship between the intrinsic matter overdensity, $\delta_{1}$, and the more observationally motivated galaxy number, $N_{\text {gal }}$ we present Figure 2 here. It shows the median overdensity of $1 h^{-1} \mathrm{Mpc}$ spheres as a function of the galaxy number in the same region. For each galaxy, we both measure $\delta_{1}$ and count the number of galaxies with mass above $10^{9.5} M_{\odot} / h$ contained in the same volume. As Figure 2 shows, once a handful of galaxies are present, the galaxy number density is linearly related to the matter overdensity. The curves of the three sample groups converge as the number of galaxies contained in the volume increases. Divergences appear when $N_{\text {gal }}$ is less than 10 . For a region with low galaxy number density, its corresponding overdensity tends to be higher if it belongs to the cluster sample compared with those which belong to the vicinity and field samples. Within our cluster galaxy sample essentially no low overdensity environment exists. This skews the distribution of galaxy number because although sometimes cluster galaxies live in relative isolation this environment is not underdense. Additionally, within this environment, as well as the matter density potentially being increased by the presence of a large dark-matter halo, gas is

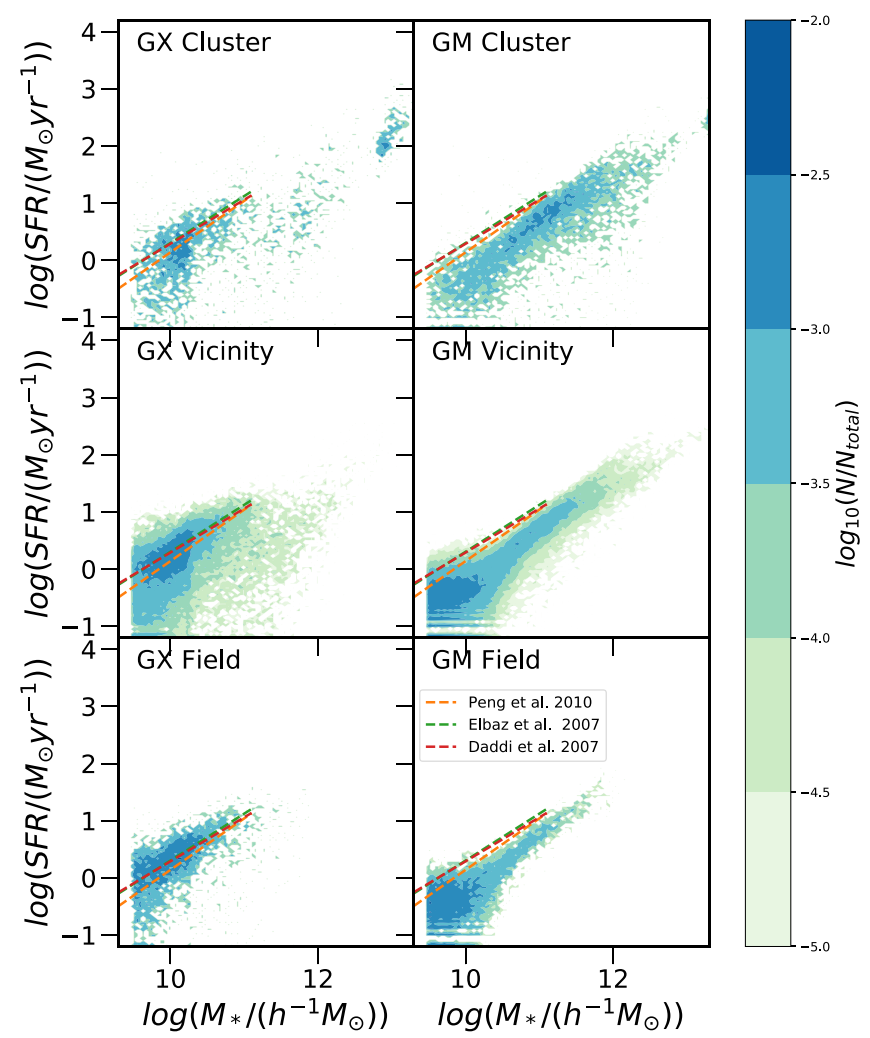

Figure 3. Star formation rate of galaxies as a function of galaxy stellar mass at $z=0$. The left column shows results for the GADGET-X simulations, the right column shows results for the GADGET-MUSIC simulations. The rows show our three environments, as indicated in the panel. The red, green, and black dashed lines indicate observational fits to the SFR $-M_{*}$ relation taken from Peng et al. (2010), Elbaz et al. (2007) and Daddi et al. (2007). According to the references, these fitting lines are valid for a rough range of $10^{7} M_{\odot}<M_{*}<10^{11} M_{\odot}$. The color shading denotes galaxy number density within the sample, as shown via the color bar on the right.

readily ram-pressure stripped. Thus at the edge of the cluster less gas is available to make stars and consequently fewer (and smaller) galaxies might be formed. Primarily though, out to twice $R_{200}$ matter overdensities much below 30 times the cosmic mean simply do not occur, and this distorts the distribution at low galaxy number.

As Figure 2 illustrates, for any single galaxy there is quite a bit of scatter in the $\delta_{1}$ to $N_{\text {gal }}$ relationship. Generally though, these two quantities are clearly related and we can use the intrinsic quantity $\delta_{1}$ interchangeably with the observational measure $N_{\text {gal }}$ in what follows. In practice, any observational measure of $N_{\text {gal }}$ would use a different mass limit for the included galaxies and so the correspondence would need to be recalculated for this value. In this case though it is $\delta_{1}$ that is the fixed quantity, with $N_{\text {gal }}$ changing.

\section{Results}

\subsection{Specific Star Formation Rate versus Environmental Overdensity}

Although in this work we are principally interested in the relationship between a galaxy's properties and the environment it lives in, we first check that a fundamental observed relation, in this case the star formation rate to stellar mass (SFR- $M_{*}$ ) relation, is recovered. In Figure 3, we show the SFR- $M_{*}$ relation at $z=0$ for our three galaxy samples, with cluster 
galaxies on the top row, vicinity galaxies in the middle and field galaxies at the bottom. The left column of panels are for galaxies within the GADGET-X models, the right column for galaxies from the GADGET-MUSIC simulations. The shading indicates galaxy density as given by the color bar. We overplot best-fit lines for $\operatorname{SFR}\left(M_{*}\right)$ derived from Daddi et al. (2007), Elbaz et al. (2007) and Peng et al. (2010). As Figure 3 shows, the GADGET-X runs reproduce galaxies with a $\mathrm{SFR}-M *$ relation in good agreement with observations, within the stellar mass range of $10^{9.5}$ to $10^{11} h^{-1} M_{\odot}$. For galaxies more massive than $10^{11} h^{-1} M_{\odot}$ in GADGET-X clusters, the majority are below the observational fits. The SFR in the high mass range is supposed to be suppressed by AGN feedback. A similar suppression may be present in the observations (e.g., see Figure 17 of Brinchmann et al. 2004).

The GADGET-X field galaxies possibly show a slightly higher SFR than the fits but this may just reflect an apparent absence of galaxies with low star formation rates within this sample. Note that the main sequence lines from observations are based on samples which are mixtures of galaxies from all our samples, while cluster and vicinity galaxies dominate our total sample. In contrast to the reasonable looking GADGET-X galaxies, the GADGET-MUSIC galaxies have an obviously lower SFR at a given mass than the observed relations. The reason for this low SFR is somewhat counterintuitive in that within the GADGET-MUSIC simulations too many stars have been formed. However, for any given galaxy the measured SFR is always low, even at higher redshift. The contradiction is only apparent: for a matched object between the two simulations the SFR is higher in the GADGET-MUSIC simulation than in the GADGET$\mathrm{X}$ simulation, so the matched galaxy acquires a larger stellar mass. This moves it to the right in Figure 3, giving it an apparently low SFR for a galaxy of this mass. Essentially, Figure 3 demonstrates that the GADGET-MUSIC simulations produce overly massive galaxies on all scales that are significantly more massive than those observed. Because of this for the remainder of this paper we concentrate our results on the more physically reasonable galaxies found within the GADGET-X simulations.

As a further check we show the evolution of the cosmic star formation rate density (SFRD) and median specific star formation rate in Figure 4. In the top panel, the star formation rate density in the field (green line) and cluster resimulations (blue line) span the observational fit of Behroozi et al. (2013) (black line). This is to be expected as the cluster re-simulation region by design contains a large cluster and many galaxies while the field region is devoid of any large clusters and contains fewer galaxies than average per unit volume. We have combined the cluster and vicinity galaxy samples into the same blue line as the spatial split between the two makes it complicated to determine the exact volume. To construct this volume at higher redshift we always choose a sphere of co-moving radius $10 h^{-1} \mathrm{Mpc}$ centered on the middle of the re-simulation volume. In common with the observations, both the cluster and field SFRD curves rise to a peak at intermediate redshift and then the SFRD falls by around an order of magnitude by $z=0$.

The evolution of the median sSFR for each of our samples is given in the lower panel of Figure 4. At $z=0$, galaxies in the field have the highest median SSFR, and cluster galaxies have the lowest median sSFR. The difference among galaxy classes is distinguishable but with large error bars. The difference becomes smaller at higher redshifts. The three median sSFR lines finally converge at about $z \geqslant 5$. The simulated sSFR

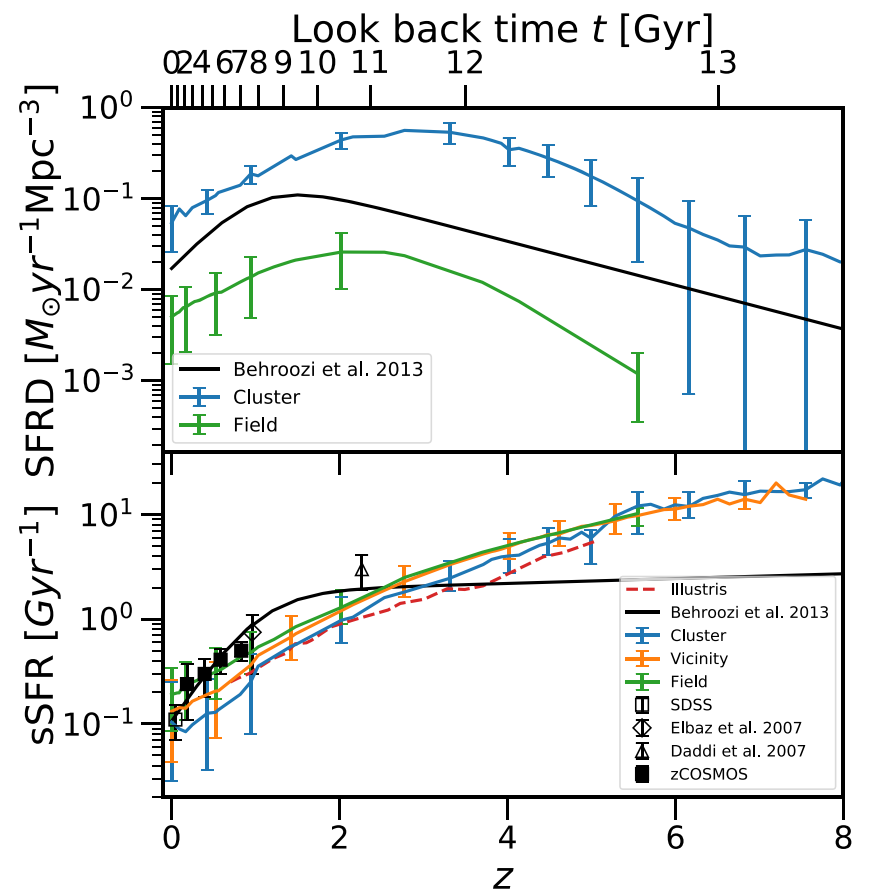

Figure 4. Evolution of the cosmic star formation rate density (SFRD, top panel) and median specific star formation rate (bottom panel). Above $z=0$, the geometry of the region defining the cluster sample is not simple, so we combine the cluster and vicinity galaxy samples. The bottom panel shows the evolution of the median specific star formation rate of galaxies with $0.8 \times 10^{10} M_{\odot} / h<M_{*}<1.2 \times 10^{10} M_{\odot} / h$. The error bars indicate the 16 th and 84 th percentile in each redshift bin. Results from different galaxy samples are distinguished by colors. The symbols with error bars are observational data derived from Figure 3 of Peng et al. (2010). In both panels, fits to observation data from Behroozi et al. (2013) are plotted as black lines. The sSFR history from the ILLUSTRIS simulation (Sparre et al. 2015) is plotted as a red dashed line in the bottom panel.

follows the observational results given by the black symbols at very low redshift, then keeps below the observational symbols from $z \sim 0.1$ to $z \sim 2.5$ before exceeding the fitting line at high redshifts. The line for cluster galaxies is not within the error bars of black observational data points, but this is reasonable because the observations take all classes of galaxies into account. In common with other hydrodynamical simulations e.g., EAGLE (Furlong et al. 2015), ILLUSTRIS (Sparre et al. 2015) and semi-analytic models, (Henriques et al. 2015), we do not recover the double-power-law fit indicated by the fit of Behroozi et al. (2013) (see Figure 5 in Davidzon et al. 2017). This lack of any clear break in the median SSFR either indicates that the observational sample is as yet incomplete or that the models are too efficient at forming stars at early times (Asquith et al. 2018).

As we have also shown above in Figure 3, many previous works have indicated a tight relationship between SFR and $M_{*}$ with $M_{*}$ expected to be $\propto \mathrm{SFR}$, from both theoretical and observational standpoints (e.g., Davé 2008; Yates et al. 2012; Wang et al. 2013). We can therefore emphasize any deviation from this relationship by calculating the specific star formation rate $\mathrm{sSFR}=\mathrm{SFR}$ gal $/ M_{*}$, which removes the direct element of the mass dependence.

In Figure 5, we show the relationship between the sSFR of our GADGET-X galaxies and the environmental overdensity around them at redshift $z=0$. We follow Steinborn et al. (2015) in using a specific star formation rate threshold of $\mathrm{sSFR}=0.3 / t_{\text {Hubble }(z)}$ to distinguish between quiescent and star-forming galaxies. 

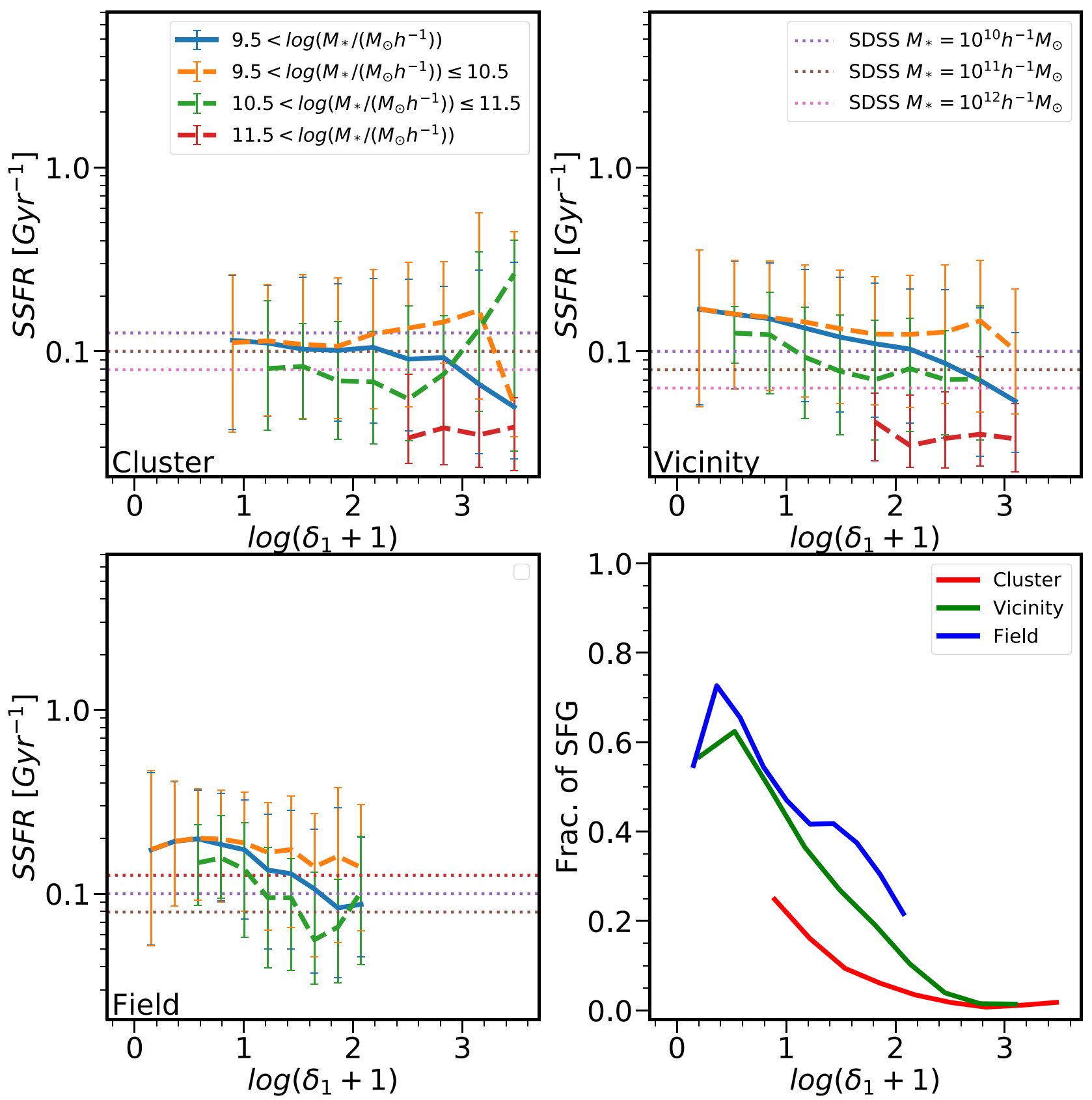

Figure 5. Median specific star formation rate (sSFR) of star-forming galaxies (SFG) for all galaxies above $10^{9.5} h^{-1} M_{\odot}$ (blue solid line) and at a range of different stellar masses within each of our three environments as indicated. The fraction of SFG within each environment is given in the lower right panel, as a function of environmental overdensity $\delta_{1}$ at redshift $z=0$. The error bars show the 16th and 84th percentiles of the samples. We also plot the fitted value of sSFR for star-forming galaxies within SDSS data for different stellar mass bins as indicated (dotted horizon lines), which are derived from Brinchmann et al. (2004).

Although this definition of a star-forming galaxy is somewhat different from the various observational criteria (see. Peng et al. 2010; Gabor \& Davé 2012; Muzzin et al. 2013), the precise division line between active and quenched galaxies varies among observations. As our simulations are not specifically tuned to match the observed sSFR- $M_{*}$ relation, we adopt a simple definition for clarity.

In all three of our samples the median sSFR for the all starforming galaxies (shown as the solid blue line) drops as the overdensity, $\delta_{1}$, increases. This is principally driven by the changing proportions of galaxies of different stellar masses. In all cases more massive galaxies have a lower sSFR and these objects tend to lie preferentially within volumes of greater local overdensity. This transition between a galaxy population at low overdensity that is dominated by small, relatively high sSFR galaxies to one at high overdensity that has a significant population of massive, low sSFR galaxies leads to the falling trend for the sample as a whole. This same drop in SSFR is also clearly seen in the observations.

We claim that the falling SSFR is principally driven by the change with mass and that the relative proportions of galaxies with different masses varies with $\delta$. This is supported by the fact that if we narrow the mass range of galaxies contributing to each of the samples shown in Figure 5 the measured sSFR becomes shallower. i.e., for broad mass bands, we again see a declining slope in the sSFR- $\delta$ relation. 
We also clearly see (Figure 5, bottom right panel) that the fraction of star-forming galaxies within each environment is also falling rapidly as the overdensity increases, with very few star-forming galaxies above overdensities of 100 in any of the samples. In the cluster sample this drop in the number of starforming galaxies appears at lower overdensities, presumably due to the contamination of this region by back splash galaxies that have passed through the cluster environment and been quenched due to gas stripping. Another feature of this panel is that the fraction of star-forming galaxies actually falls at low overdensity in both the field and vicinity galaxy samples. Such low overdensities simply do not exist in the cluster environment and so no galaxies can possibly reside there. We check components of quenched galaxies in the lowest density bin and find that most of them (over 90\%) are gas rich. There is no mechanism of specially heating the gas in low-density regions in our simulations. Combining with the fact that the galaxy number in the lowest density bin is ten times less than that in the secondary lowest density bin, we consider the falling a reasonable fluctuation rather than physical phenomenon.

For galaxies within a specified stellar mass range our simulations indicate that the sSFR tends to be rather flat, particularly in the cluster and cluster vicinities samples. In the field the sSFR for the intermediate mass range appears to fall as the overdensity increases. The apparent constancy is consistent with previous observations (e.g., Peng et al. 2010). The amplitude of the sSFR is also approximately recovered in all environments.

In Figure 5, the flat environmental dependencies of median sSFR and the falling fraction of SFG seem to be at odds with each other as the median SSFR of star-forming galaxies is not suppressed at higher environmental densities while a higher fraction of galaxies are quenched. This reproduces the results found by Peng et al. (2010). Peng et al. (2010) suggested a sharp transition scenario: galaxies continue forming stars at the same rate regardless of their environment, despite the fact that the chance of having been quenched evidently does depend strongly on the environment. This scenario requires instantaneous quenching to occur, and this is the assumption Peng et al. (2010) made in their work. While such instantaneous quenching might appear idealistic, our simulations support this scenario. Examining star formation histories for individual galaxies we often see sharp drops in SFR during a galaxy's lifetime. Other work suggests that galaxies do not undergo an instantaneous quenching scenario. Using subhalo abundance matching (SHAM), Wetzel et al. (2013), Muzzin et al. (2014) suggested rapid quenching for satellite galaxies with a quenching timescale of less than 0.8 Gyr. Hahn et al. (2017) suggested a longer quenching timescale of $0.5-1.5$ Gyr for central galaxies. Currently observational evidence on quenching timescales is lacking, and we therefore cannot confirm which hypothesis truly describes a galaxy's SFR evolution.

We further investigate how the median sSFR evolves with redshift in Figure 6, with different line styles indicating our three different samples. All galaxies with stellar mass above $10^{9.5} h^{-1} M_{\odot}$ are included. Figure 6 shows that the fraction of SFG versus $\delta_{1}$ (top panel) and sSFR $-\delta_{1}$ (bottom panel) retain a similar shape at all redshifts, with the amplitude decreasing with time. At earlier times three classes of galaxies are in agreement with each other. It is because that at that time the environments are not obviously distinguishable. Essentially, there is a higher fraction of SFG at early times and these

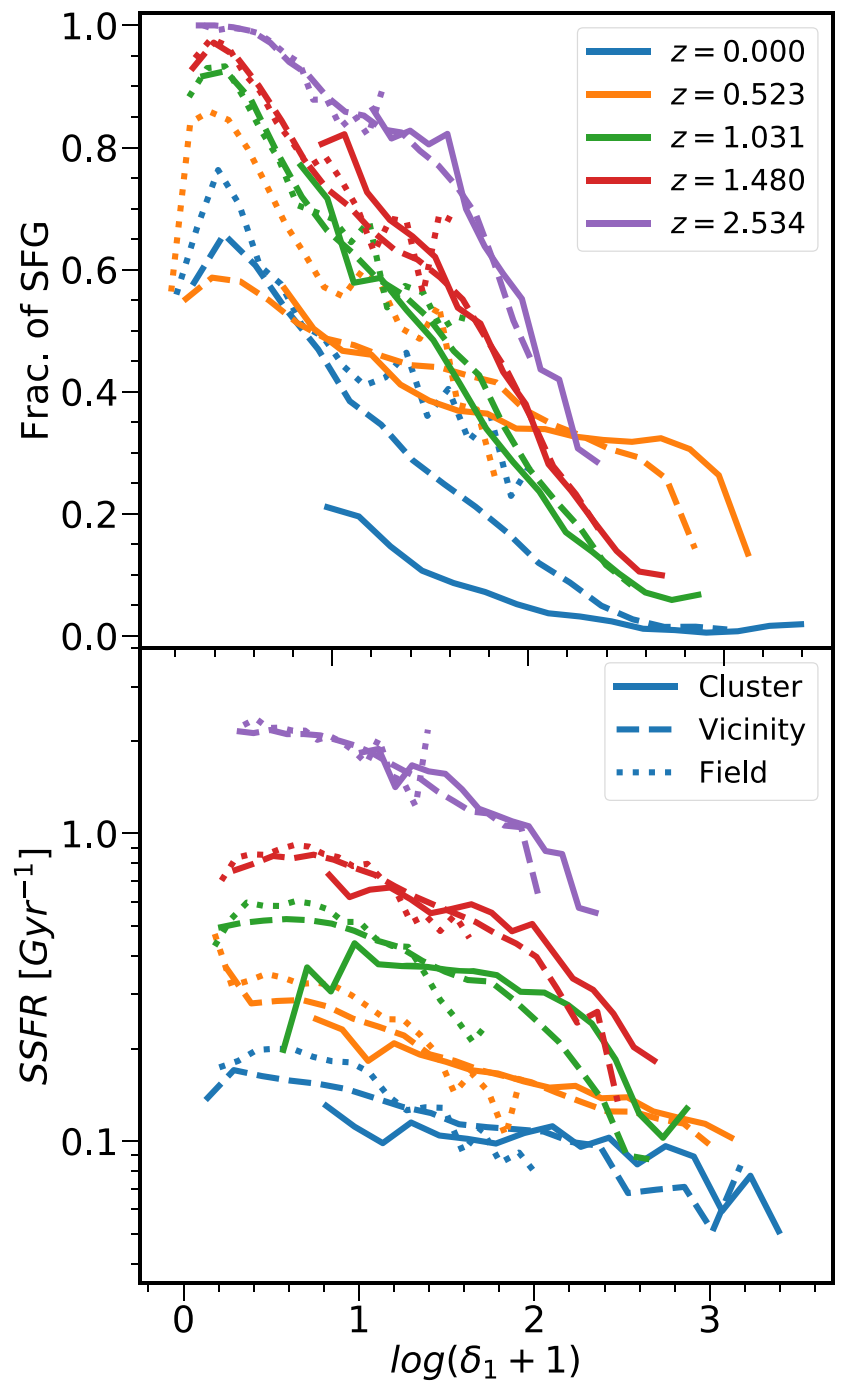

Figure 6. Fraction of star-forming galaxies (top panel) and median sSFR of starforming galaxy as functions of environmental overdensity $\delta_{1}$ (bottom panel) at various different redshifts as indicated. Galaxies with stellar mass $M_{*}>10^{9.5} h^{-1} M_{\odot}$ are included. Lines for different redshifts are distinguished with different colors, with different environmental sample groups distinguished via line style. The redshift zero lines are reproduced from Figure 5 for comparison.

galaxies are in general forming stars at a faster rate. There is no sign of any difference between three galaxy classes, but there is some evidence that the dependence of sSFR on overdensity somewhat steepens at early times.

While our simulations appear to do a reasonable job at reproducing many of the features of the observed sSFR- $\delta_{1}$ relationship we re-iterate that our simulations were not tuned to fit this, although many properties are consistent with observations (see. Rasia et al. 2015; Biffi et al. 2017, 2018; Truong et al. 2018). One issue is the diversity among observational claims. Patel et al. $(2009,2011)$ found a declining trend for the sSFR-local density relation of star-forming galaxies at $0.6<z<0.9$ and Muzzin et al. (2012) found the sSFR of star-forming galaxies increases with the distance from the cluster center at $z \sim 1$. These results are consistent with our work. However, there are also works which show no correlation between these properties at high redshift: Grützbauch et al. (2011), Scoville et al. (2013) did not found any correlation between the sSFR of all galaxies and the local 


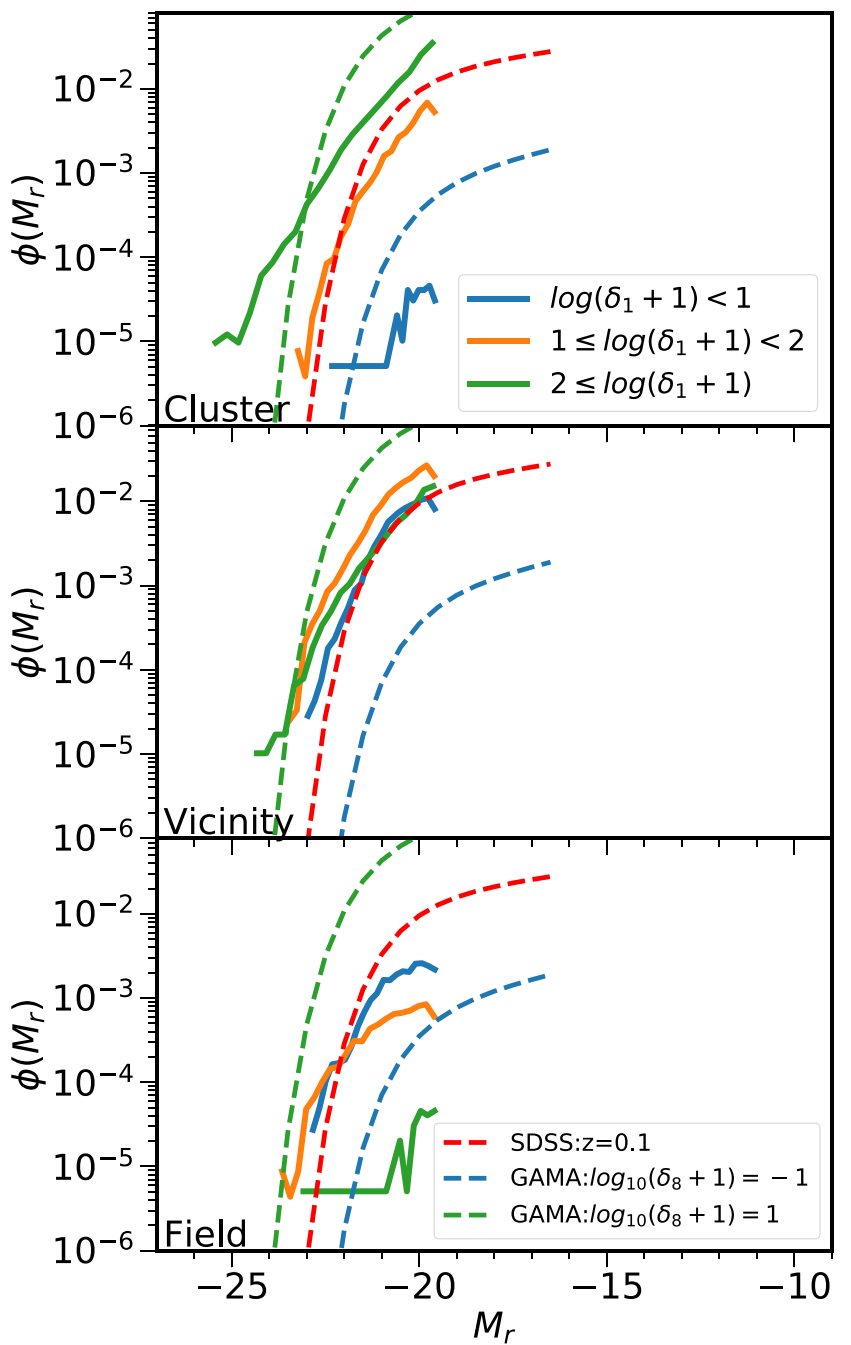

Figure 7. $r$ band luminosity function of galaxies for three bands of environmental overdensity for each of our three galaxy samples. As reference, the ${ }^{0.1} r$ - band SDSS DR6 luminosity function is shown via the red dashed line. Two best-fit lines indicating the luminosity function of underdense (dashed blue) and overdense (dashed green) galaxies within the GAMA survey are derived from McNaught-Roberts et al. (2014).

overdensity at $z>1$. Darvish et al. (2016) found that the SFR and sSFR of star-forming galaxies are not related to the environmental overdensity from $z=0.1$ to $z=3$. As Darvish et al. (2016) claimed, the result that no dependence on environment is found at $z \geqslant 1$ may be partly due to the larger photo- $z$ uncertainties at higher redshift and the lack of extremely dense regions in the COSMOS field. Moreover, Elbaz et al. (2007), Cooper et al. (2008), Welikala et al. (2016) found an ascending trend of the SFR-density relation at high redshift, which is opposite to what Patel et al. (2009) found or other SFR-density relations at low redshifts. Popesso et al. (2011) attributed such a reversal in the relation to the contamination of AGN.

\subsection{Luminosity and Color versus Environmental Overdensity}

First we present the $r$ band luminosity function for three bands of environmental overdensity, $\delta_{1}$ for each of our three different sample groups in Figure 7. The Luminosity function for galaxies within a specific density bin is measured following the method described in Section 2.5 of (McNaught-Roberts et al. 2014)

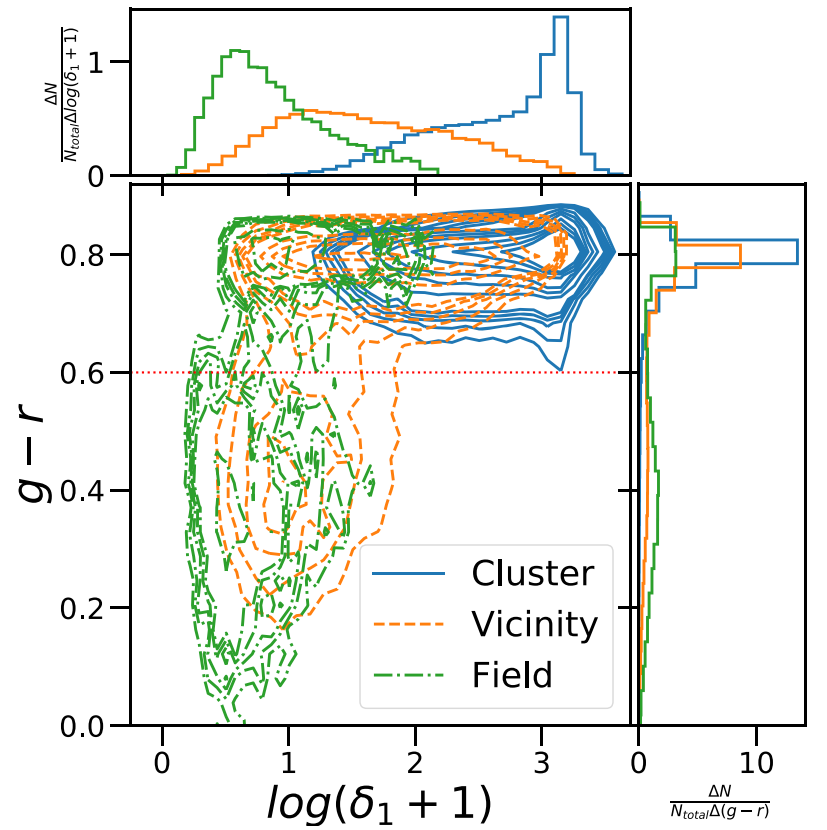

Figure 8. In the main panel, the contours show the number density of galaxies on the $g-r$ vs. $\delta_{1}$ plane at redshift $z=0$. Contours for each of our three sample groups are distinguished by different line colors and styles. The horizontal red dotted line at $g-r=0.6$ indicates the rough division between red and blue galaxies at redshift $z=0$. In the top and right subplots, the histograms show the galaxy number density in each overdensity/color bin again split by galaxy sample.

The dashed lines indicate best fits to the SDSS luminosity function (red) and galaxies within under- (blue) and over- (green) dense environments within the GAMA survey. The green solid lines in each panel indicate galaxies with the highest local overdensity, $\delta_{1}$. There are lots of these in the cluster sample and they are generally brighter and more massive than the galaxies in less overdense environments. Even though we have removed the central galaxies from each of the 324 clusters (these are hard to distinguish from their surrounding intracluster light) there remain some extremely bright objects. This indicates that the treatment of large central galaxies within the GADGET-X model could be improved. The excess here is due to central galaxies within large infalling structures. This effect for the most overdense galaxies persists into the vicinities (middle panel) and occurs for the same reason: there are some large groups with dominant central galaxies here. In the field sample there are no large groups by construction and so there are very few galaxies in the most overdense environment. For the orange and blue solid lines indicating galaxies in intermediate and low overdensity local environments the luminosity function in all three of our sample regions is not unrealistic when compared with the observational best fits. The shape is well recovered in all cases with, as expected, the amplitude of the underdense luminosity function rising as we move from the cluster to vicinities to field samples. The observed luminosity function is built up from galaxies within all three of our sample regions and is a combination of them with unknown weights. Essentially, unless a very large unbiased survey has been constructed the precise amplitude of the luminosity function curves will depend strongly on the sample selection.

Second, we check the color $-\delta_{1}$ relation in Figure 8 . We use the difference between the magnitude in the $g$ and $r$ bands, 


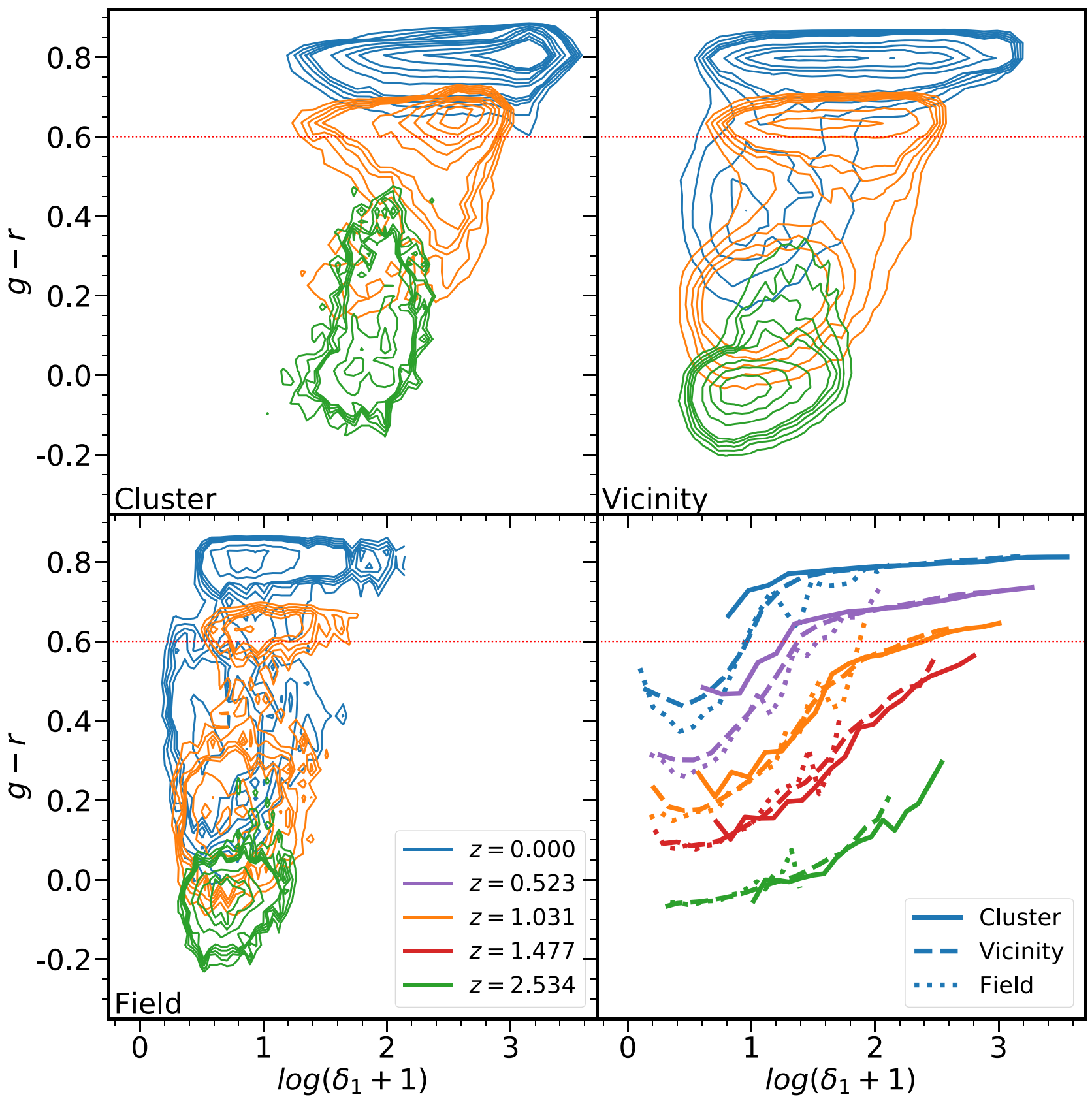

Figure 9. Contours of galaxy number density on the $g-r$ vs. $\delta_{1}$ plane at different redshifts as indicated by the various colors. Three of the panels present the results from each of our three sample groups as indicated. For brevity, only contours at $z=0, z=1.031$ and $z=2.534$ are shown. The bottom right-hand panel presents the median relationship between $g-r$ and $\delta_{1}$ split via line style for each sample group and by color as a function of redshift. The horizontal red dotted line at $g-r=0.6$ indicates the rough division between red and blue galaxies at redshift $z=0$.

$M_{g}-M_{r}$ to evaluate the color of each galaxy and display this as a function of overdensity, $\delta_{1}$. Contours of different colors indicate the galaxy number density at $z=0$ within each of our three sample regions as indicated in the legend. The horizontal dotted red line at $g-r=0.6$ indicates the rough division between red and blue galaxies at redshift $z=0$. Clearly, within the cluster sample the vast majority of the galaxies are red at $z=0$, with almost no blue galaxies. There are also no galaxies with low environmental overdensity because such an environment does not exist for this sample. The field sample contains examples of both red and blue galaxies and, as previously seen, does not contain any high overdensity environment (and therefore has no high overdensity galaxies). The cluster vicinities sample contains galaxies with both colors and environments, although it does not probe either the highest or the lowest overdensities. The clear bimodality between blue and red galaxies in the observed galactic population is well recovered by the GADGET-X simulations.

Finally we examine the evolution of the color $-\delta_{1}$ plane in Figure 9. Three panels show via colored contours the change in the distribution of galaxy number density on the $g-r$ versus $\delta_{1}$ plane as a function of redshift. Each panel displays results from a different sample region as indicated. As before, the red dotted horizontal line at $g-r=0.6$ indicates the rough division between red and blue galaxies at redshift $z=0$. Clear evolution in the color of the galactic population in all three 
samples is seen and this mirrors the expected trend. In all three samples an obvious red sequence is visible and in-place at $z=1$ and color bimodality is also evident. At $z \sim 2.5$ the majority of galaxies in all environments are blue, even if the evolution of the dividing line between red and blue galaxies is taken to evolve with redshift. The bottom right panel displays the evolution of the median values within each sample (distinguished via line type) as a function of redshift (distinguished via line color). The median color as a function of overdensity within all three of our samples is largely indistinguishable, indicating that to a large extent the apparent differences in color as a function of $\delta$ are largely driven by how well the overdensity range is spanned by the sample rather than via any difference in the galaxy's color due to its environment.

\subsection{Local Overdensity versus Halo Mass}

One critical issue is what environmental measure is most closely related to a galaxy's properties. Based on the theory of hierarchical structure formation, it is quite natural to think that a galaxy's properties should be closely linked to their host virialized dark-matter halo. On the other hand, many observations found a close relationship between local overdensity and galactic properties. Our simulations support the observational result, as we find that local overdensity is a more direct measure of a galaxy's properties than its host halo mass.

An intuitive way to quantify the relationship between two variables is by calculating Spearman's rank correlation coefficient. Spearman's rank correlation coefficient $r_{s}$ is defined as

$$
r_{s}=\frac{\operatorname{cov}\left(r g_{X}, r g_{Y}\right)}{\sigma_{r g_{X}} \sigma_{r g_{Y}}} .
$$

Here, $r g_{X i}$ is the rank of $i$ th element in the array of variable $X$. The value of $r_{s}$ indicates how well the relationship between two variables can be described using a monotonic function. The range of $r_{s}$ is $[-1,1], r_{s}=0$ indicating no relationship between the two variables. $r_{s}=1$ indicates a perfect positive relationship, while $r_{s}=-1$ indicates a perfect negative relationship. The larger the value of $\left|r_{s}\right|$, the better the relationship is.

In Table 2, we tabulate the value of $r_{s}$ between galaxy properties and three environmental measures, namely $\delta_{1}$, the host halo mass and the galaxy stellar mass. We calculate this measure for all three of our galaxy samples, as indicated in the table. The value of $r_{s}\left(\delta_{1}\right)$ is quite consistent among our three samples. As we have already seen above: the fraction of SFG is strongly negatively correlated with $\delta_{1}$; the sSFR of SFG is slightly negatively correlated with $\delta_{1}$; the color, $g-r$, is positively correlated with $\delta_{1}$. The $r_{s}\left(M_{\text {halo }}\right)$ shows a quite different relationship, as well as changing significantly across the three galaxy samples. This variation in $r_{s}\left(M_{\text {halo }}\right)$ implies that the influence of host halo differs in different large-scale environments. Thus halo mass is a worse indicator of local environment than local overdensity $\delta_{1}$. This should not necessarily be a surprise, as a large galaxy cluster contains many thousands of galaxies with a wide variation in properties and so the single halo mass should not be expected to correlate particularly well on this level.

For comparison we also calculate Spearman's correlation coefficient for galaxy properties and stellar mass. In this case, for the cluster sample, $r_{s}$ has a value of 0.924 for the fraction of star-forming galaxies, which indicates a significant positive
Table 2

Spearman's Correlation Coefficient $r_{s}$ for the Relationship between Galaxy Properties and Local Overdensity, Halo Mass, and Stellar Mass

\begin{tabular}{lrrr}
\hline \hline Cluster & & & \\
\hline$r_{s}$ & Frac $_{\mathrm{SFG}}$ & sSFR $_{\mathrm{SFG}}$ & $g-r$ \\
\hline$\delta_{1}$ & -0.932 & -0.099 & 0.311 \\
$M_{\text {halo }}$ & 0.809 & -0.280 & -0.104 \\
$M_{*}$ & 0.924 & -0.321 & 0.388 \\
\hline
\end{tabular}

Vicinity

\begin{tabular}{lrrr}
\hline$r_{s}$ & Frac $_{\mathrm{SFG}}$ & sSFR $_{\mathrm{SFG}}$ & $g-r$ \\
\hline$\delta_{1}$ & -0.962 & -0.161 & 0.454 \\
$M_{\text {halo }}$ & 0.528 & -0.041 & 0.009 \\
$M_{*}$ & -0.364 & -0.209 & 0.398 \\
\hline
\end{tabular}

Field

\begin{tabular}{lrrr}
\hline$r_{s}$ & Frac $_{\mathrm{SFG}}$ & sSFR $_{\mathrm{SFG}}$ & $g-r$ \\
\hline$\delta_{1}$ & -0.946 & -0.178 & 0.373 \\
$M_{\text {halo }}$ & 0.502 & -0.111 & -0.293 \\
$M_{*}$ & -0.490 & -0.250 & 0.421 \\
\hline
\end{tabular}

Note. $r_{s}$ for our cluster, vicinity, and field galaxy samples are listed in separate tables.

relationship. This relationship is opposite to that observed due to the essential lack of low-mass SFG in the cluster sample. Except for this, the values of the other $r_{s}\left(M_{*}\right)$ meet our expectations well. By comparing $r_{s}\left(M_{*}\right)$ and $r_{s}\left(\delta_{1}\right)$, we find that the fraction of SFG is more influenced by the local overdensity, while the sSFR of SFG is more influenced by the stellar mass. For galaxy color, it seems that both $\delta_{1}$ and $M_{*}$ have similar weights.

The accuracy of $r_{s}$ could suffer due to the incompleteness of our samples. Thus we divided our galaxies into different stellar mass bins to test the sensitivity of $r_{s}$ to our galaxy sample. As Table 3 shows, the $r_{s}$ for the low-mass and high mass ends changes a lot compared with Table 2, while the $r_{s}$ for the median stellar mass range keeps the same trends and similar values. Except for massive galaxies, the strong influence of environment, $\delta_{1}$, on the fraction of SFG is stable, which further prove the important link between environment and the SFG fraction. The negative correlation between $M_{*}$ and sSFR of SFG is still clear, although it becomes weaker in the low and high stellar mass range. The $r_{s}\left(M_{\text {halo }}\right)$ becomes variable when the different stellar mass ranges are applied. The $r_{s}$ between color $g-r$ and $\delta_{1}, M_{*}, M_{\text {halo }}$ is very similar to Table 2, except for massive galaxies. $r_{s}\left(M_{\text {halo }}, g-r\right)$ shows negative relation in most places, which is again counterintuitive. In summary, we consider that the influence of $\delta_{1}$ is much less sensitive to mass completeness than $M_{\text {halo }}$.

\section{Discussion and Conclusions}

In this work, we have examined the local environmental dependence, measured via the overdensity relative to the cosmic mean on a scale of $1 \mathrm{Mpc} / h, \delta_{1}$, of several galactic properties. We do this with three galaxy samples which broadly delineate the large-scale environment, specifically a field sample constructed from four field resimulations of volumes that do not contain any large halo at $z=0$, a cluster sample that contains all galaxies within twice $R_{200}$ of the center of the 
Table 3

The Same as Table 2, But the Samples are Divided into three Stellar Mass Bins

\begin{tabular}{|c|c|c|c|c|c|c|c|c|c|}
\hline$\frac{\text { Cluster }}{r_{s}}$ & \multicolumn{3}{|c|}{$M_{*}<10^{10} h^{-1} M_{\odot}$} & \multicolumn{3}{|c|}{$10^{10} h^{-1} M_{\odot}<M_{*}<10^{11.5} h^{-1} M_{\odot}$} & \multicolumn{3}{|c|}{$M_{*}>10^{11.5} h^{-1} M_{\odot}$} \\
\hline$\delta_{1}$ & -0.883 & 0.082 & 0.446 & -0.933 & 0.076 & 0.326 & 0.939 & -0.013 & -0.400 \\
\hline$\underline{M_{*}}$ & 0.818 & -0.076 & 0.283 & 0.552 & -0.143 & 0.102 & 0.564 & -0.095 & -0.731 \\
\hline Vicinity & \multicolumn{3}{|c|}{$M_{*}<10^{10} h^{-1} M_{\odot}$} & \multicolumn{3}{|c|}{$10^{10} h^{-1} M_{\odot}<M_{*}<10^{11.5} h^{-1} M_{\odot}$} & \multicolumn{3}{|c|}{$M_{*}>10^{11.5} h^{-1} M_{\odot}$} \\
\hline$\delta_{1}$ & -0.964 & -0.080 & 0.638 & -1.0 & -0.171 & 0.396 & 0.127 & -0.026 & -0.456 \\
\hline$M_{\text {halo }}$ & 0.818 & 0.134 & -0.750 & 0.152 & -0.025 & -0.088 & 0.033 & -0.106 & -0.458 \\
\hline$M_{*}$ & $\cdots$ & -0.082 & 0.013 & -0.685 & -0.199 & 0.169 & 0.762 & 0.033 & -0.499 \\
\hline Field & \multicolumn{3}{|c|}{$M_{*}<10^{10} h^{-1} M_{\odot}$} & \multicolumn{3}{|c|}{$10^{10} h^{-1} M_{\odot}<M_{*}<10^{11.5} h^{-1} M_{\odot}$} & \multicolumn{3}{|c|}{$M_{*}>10^{11.5} h^{-1} M_{\odot}$} \\
\hline$r_{s}$ & Frac $_{\mathrm{SFG}}$ & $\mathrm{sSFR}_{\mathrm{SFG}}$ & $g-r$ & Frac $_{\mathrm{SFG}}$ & $\mathrm{sSFR}_{\mathrm{SFG}}$ & $g-r$ & Frac $_{\mathrm{SFG}}$ & $\mathrm{sSFR}_{\mathrm{SFG}}$ & $g-r$ \\
\hline
\end{tabular}

largest halo within each of our 324 cluster resimulations and a "vicinity" sample that consists of all those galaxies not in the cluster sample but within the uncontaminated region of the cluster resimulations. These latter vicinity galaxies are by definition relatively close to a large structure. Cluster galaxies are essentially within a large structure (the median mass of the our cluster halos is $8 \times 10^{14} M_{\odot} / h$ ). Field galaxies are a long way from any major dark-matter halo, with the largest halo contained within them having a mass of $4 \times 10^{13} M_{\odot} / h$.

One primary advantage of our project "THE THREE HUNDRED" (Cui et al. 2018) is the abundance of simulated galaxies. Because we in total employed 328 resimulations, the number of available galaxies at any one time exceed 200,000, which is almost at the same level as observations, i.e., Peng et al. (2015) select 238,474 redshift reliable galaxies from SDSS DR7 for analysis. Such an abundance of galaxies allow us to test the dependence of galaxy properties on environment convincingly, as well as other relations. In addition, we employ an advanced hydrodynamical code GADGET-X which reasonably recover the observed galactic $\mathrm{SFR}-M_{*}$ relation.

There are three main environmental relations explored in this work:

1. First, the environmental overdensity $\delta_{1}$ has no influence on the measured specific star formation rate of starforming galaxies. Although, the sSFR for all galaxies does show a trend with overdensity, this is driven by the changing balance of galactic mass with environment. At fixed stellar mass, the sSFR is independent of the local overdensity. There is a larger fraction of massive galaxies in overdense environments than in underdense environments, and the sSFR is clearly dependent on the mean stellar mass of the sample. This mirrors the observational result found by SDSS, where the sSFR also drops as the median galaxy mass increases.

2. Second, as expected, the fraction of star-forming galaxies declines as the overdensity increases in all three of our samples. Our three classes of galaxies have different SFG Fraction- $\delta_{1}$ relation at $z=0$, but all three curves converge at high redshift. This implies that, in spite of local overdensity, the large-scale structure, such as the presence of a cluster or a void, affects the quenching process. At high redshift, these large-scale structures have not formed and thus their influence does not yet show up.

3. Third, galaxies become redder in higher overdensity environments. The color distribution of galaxies with overdensity is bimodal as expected, with a clear red sequence and blue cloud. Median colors as a function of overdensity compared in the field, vicinity, and cluster samples do not change. This is somewhat surprising given the visual impression of the contour plots. What is actually happening is that different regions of the overdensity axis are being probed, but the underlying distribution is largely unchanged.

We further qualified three environmental relations above with Spearman's correlation coefficient $r_{s}$. The value of $r_{s}$ for each galaxy property- $\delta_{1}$ relation is in agreement with what we have seen in the figures. Spearman's correlation coefficient $r_{s}$ for galaxy properties and both $\delta_{1}$ and $M_{\text {halo }}$ is also compared. We found that $r_{s}$ for the relationship between $\delta_{1}$ and galaxy properties is much more stable than the relationship with halo mass. This implies that, compared with halo mass, local overdensity is more likely to be directly linked to a galaxy's properties. We stress that Spearman's correlation coefficient $r_{s}$ is a parameter evaluating the monotonic relation. It is possible that the relationship between a galaxy's properties and host halo mass is not monotonic.

Our galaxy sample recovers many observational properties well, but it is not yet perfect. Within the set of GADGET-X galaxies some are unrealistically bright. These are the dominant central objects within large dark-matter halos. Future iterations of the GADGET-X code will examine this issue further. This aside, the luminosity function of the galaxies produced by GADGET-X has the correct shape. The amplitude is dependent on the relative abundance of that particular environment within the sample and so is largely driven by sample selection. Care should be taken when constructing galaxy luminosity functions to avoid this dependence on local environment.

To summarize, the dependence of galaxy star formation rates and color on overdensity for field, vicinity, and cluster samples is examined as a function of redshift using a large sample of 
324 massive galaxy clusters. We show that the GADGET-X galaxies within these objects (and also in field regions) generally form a reliable sample which can be used to test observational inferences upon the formation and evolution of the galactic population.

This work has received financial support from the European Union's Horizon 2020 Research and Innovation programme under the Marie Sklodowskaw-Curie grant agreement number 734374 , i.e., the LACEGAL project.

The authors thank The Red Española de Supercomputación for granting us computing time at the MareNostrum Supercomputer of the BSC-CNS where most of the cluster simulations have been performed. Part of the computations with Gadget-X have also been performed at the "LeibnizRechenzentrum" with CPU time assigned to the Project "pr83li." The authors would like the acknowledge the Centre for High Performance Computing in Rosebank, Cape Town for financial support and for hosting the "Comparison Cape Town" workshop in 2016 July. The authors would further like to acknowledge the support of the International Centre for Radio Astronomy Research (ICRAR) node at the University of Western Australia (UWA) in the hosting the precursor workshop "Perth Simulated Cluster Comparison" workshop in March 2015; the financial support of the UWA Research Collaboration Award 2014 and 2015 schemes; the financial support of the ARC Centre of Excellence for All Sky Astrophysics (CAASTRO) CE110001020; and ARC Discovery Projects DP130100117 and DP140100198. We also thank the Instituto de Fisica Teorica (IFT-UAM/CSIC in Madrid) for its support, via the Centro de Excelencia Severo Ochoa Program under Grant No. SEV-2012-0249, during the three week workshop "nIFTy Cosmology" in 2014, where the foundation for this this project was established.

Y.W. is supported by the NSFC No.11643005. L.L.F. is supported by the NSFC No.11733010. W.C., A.K., and G.Y. are supported by the Ministerio de Economía y Competitividad and the Fondo Europeo de Desarrollo Regional (MINECO/ FEDER, UE) in Spain through grant AYA2015-63810-P. A.K. is further supported by the Spanish Red Consolider MultiDark FPA201790566REDC and also thanks Nohelani Cypriano for lihue.

Y.W. thanks Weishan Zhu and Jiaxin Han for their useful comments and suggestions. We also thank Giuseppe Murante for the help with running the GADGET-X simulations.

The authors contributed to this paper as follows. G.Y., F.R.P., A.K., C.P., and W.C. formed the core team that provided and organized the simulations and general analysis of data. G.Y., and S.G. ran at LRZ Munich the underlying MDPL2 simulation and selected the regions for the resimulations. A.A. and Giuseppe Murante managed the GADGET-X simulation and provided the data. The specific data analysis for this paper was led by Y.W. Y. W., F.R.P., and A.K. wrote the text. All authors had the opportunity to proofread and provide comments on the paper.

\section{ORCID iDs}

Yang Wang (10) https://orcid.org/0000-0002-1512-5653 Alexander Knebe (i) https://orcid.org/0000-0003-4066-8307 Gustavo Yepes $(10$ https://orcid.org/0000-0001-5031-7936 Weiguang Cui (1) https://orcid.org/0000-0002-2113-4863 Chris Power (i) https://orcid.org/0000-0002-4003-0904

\section{References}

Arthur, J., Pearce, F. R., Gray, M. E., et al. 2016, MNRAS, 464, 2027 Asquith, R., Pearce, F. R., Almaini, O., et al. 2018, MNRAS, 480, 1197 Beck, A. M., Murante, G., Arth, A., et al. 2016, MNRAS, 455, 2110 Behroozi, P. S., Wechsler, R. H., \& Conroy, C. 2013, ApJ, 770, 57 Biffi, V., Dolag, K., \& Merloni, A. 2018, arXiv:1804.01096 Biffi, V., Planelles, S., Borgani, S., et al. 2017, MNRAS, 468, 531 Borgani, S., \& Kravtsov, A. 2011, ASL, 4, 204

Brinchmann, J., Charlot, S., White, S. D. M., et al. 2004, MNRAS, 351, 1151 Capak, P., Abraham, R. G., Ellis, R. S., et al. 2007, ApJS, 172, 284 Chandrasekhar, S. 1943, ApJ, 97, 255

Contini, E., De Lucia, G., \& Borgani, S. 2012, MNRAS, 420, 2978

Cooper, M. C., Newman, J. A., Weiner, B. J., et al. 2008, MNRAS, 383, 1058 Cui, W., Knebe, A., Yepes, G., et al. 2018, MNRAS, 480, 2898

Cui, W., Power, C., Knebe, A., et al. 2016, MNRAS, 458, 4052

Cui, W., \& Zhang, Y. 2017, in The Impact of Baryons on the Large-Scale Structure of the Universe, ed. A. J. Capistrano de Souza (InTech Open)

Daddi, E., Dickinson, M., Morrison, G., et al. 2007, ApJ, 670, 156

Darvish, B., Mobasher, B., Sobral, D., et al. 2016, ApJ, 825, 113

Darvish, B., Sobral, D., Mobasher, B., et al. 2014, ApJ, 796, 51

Davé, R. 2008, MNRAS, 385, 147

Davidzon, I., Ilbert, O., Faisst, A. L., Sparre, M., \& Capak, P. L. 2017, arXiv: 1712.03959

De Lucia, G., Fontanot, F., \& Wilman, D. 2012a, MNRAS, 419, 1324

De Lucia, G., Weinmann, S., Poggianti, B. M., Aragón-Salamanca, A., \& Zaritsky, D. 2012b, MNRAS, 423, 1277

Devriendt, J. E. G., Guiderdoni, B., \& Sadat, R. 1999, A\&A, 350, 381 Dressler, A. 1980, ApJ, 236, 351

Elahi, P. J., Knebe, A., Pearce, F. R., et al. 2016, MNRAS, 458, 1096

Elbaz, D., Daddi, E., Le Borgne, D., et al. 2007, A\&A, 468, 33

Fabello, S., Kauffmann, G., Catinella, B., et al. 2012, MNRAS, 427, 2841

Furlong, M., Bower, R. G., Theuns, T., et al. 2015, MNRAS, 450, 4486

Gabor, J. M., \& Davé, R. 2012, MNRAS, 427, 1816

Gabor, J. M., \& Davé, R. 2015, MNRAS, 447, 374

Gallazzi, A., Bell, E. F., Wolf, C., et al. 2009, ApJ, 690, 1883

Grützbauch, R., Conselice, C. J., Bauer, A. E., et al. 2011, MNRAS, 418, 938

Gunn, J. E., \& Gott, J. R., III 1972, ApJ, 176, 1

Hahn, C., Tinker, J. L., \& Wetzel, A. 2017, ApJ, 841, 6

Hahn, O., Porciani, C., Carollo, C. M., \& Dekel, A. 2007, MNRAS, 375, 489

Hashimoto, Y., Oemler, A., Jr., Lin, H., \& Tucker, D. L. 1998, ApJ, 499, 589

Henriques, B. M. B., White, S. D. M., Thomas, P. A., et al. 2015, MNRAS, 451, 2663

Kauffmann, G., White, S. D. M., Heckman, T. M., et al. 2004, MNRAS, 353,713

Kennicutt, R. C., Jr. 1998, in ASP Conf. Ser. 142, The Stellar Initial Mass Function (38th Herstmonceux Conference), ed. G. Gilmore \& D. Howell (San Francisco, CA: ASP), 1

Klypin, A., Yepes, G., Gottlöber, S., Prada, F., \& Heß, S. 2016, MNRAS, 457, 4340

Knollmann, S. R., \& Knebe, A. 2009, ApJS, 182, 608

Kovač, K., Lilly, S. J., Cucciati, O., et al. 2010, ApJ, 708, 505

Lai, C.-C., Lin, L., Jian, H.-Y., et al. 2016, ApJ, 825, 40

McCarthy, I. G., Frenk, C. S., Font, A. S., et al. 2008, MNRAS, 383, 593

McNaught-Roberts, T., Norberg, P., Baugh, C., et al. 2014, MNRAS, 445, 2125

Mihos, J. C., \& Hernquist, L. 1994, ApJL, 437, L47

Mo, H. J., \& White, S. D. M. 1996, MNRAS, 282, 347

Moore, B., Katz, N., Lake, G., Dressler, A., \& Oemler, A. 1996, Natur, 379,613

Muldrew, S. I., Croton, D. J., Skibba, R. A., et al. 2012, MNRAS, 419, 2670

Muzzin, A., Marchesini, D., Stefanon, M., et al. 2013, ApJ, 777, 18

Muzzin, A., van der Burg, R. F. J., McGee, S. L., et al. 2014, ApJ, 796, 65

Muzzin, A., Wilson, G., Yee, H. K. C., et al. 2012, ApJ, 746, 188

Oemler, A., Jr. 1974, ApJ, 194, 1

Patel, S. G., Holden, B. P., Kelson, D. D., Illingworth, G. D., \& Franx, M. 2009, ApJL, 705, L67

Patel, S. G., Kelson, D. D., Holden, B. P., Franx, M., \& Illingworth, G. D. 2011, ApJ, 735, 53

Peng, Y., Maiolino, R., \& Cochrane, R. 2015, Natur, 521, 192

Peng, Y.-j., Lilly, S. J., Kovač, K., et al. 2010, ApJ, 721, 193

Popesso, P., Rodighiero, G., Saintonge, A., et al. 2011, A\&A, 534, C2

Quilis, V., Moore, B., \& Bower, R. 2000, Sci, 288, 1617

Rafieferantsoa, M., Davé, R., Anglés-Alcázar, D., et al. 2015, MNRAS, 453, 3980

Rasia, E., Borgani, S., Murante, G., et al. 2015, ApJL, 813, L17 
Scoville, N., Arnouts, S., Aussel, H., et al. 2013, ApJS, 206, 3

Sembolini, F., Elahi, P. J., Pearce, F. R., et al. 2016a, MNRAS, 459, 2973

Sembolini, F., Yepes, G., De Petris, M., et al. 2013, MNRAS, 429, 323

Sembolini, F., Yepes, G., Pearce, F. R., et al. 2016b, MNRAS, 457, 4063

Sparre, M., Hayward, C. C., Springel, V., et al. 2015, MNRAS, 447, 3548

Springel, V. 2005, MNRAS, 364, 1105

Springel, V., White, S. D. M., Jenkins, A., et al. 2005, Natur, 435, 629

Steinborn, L. K., Dolag, K., Hirschmann, M., Prieto, M. A., \& Remus, R.-S. 2015, MNRAS, 448, 1504

Tanaka, M., Goto, T., Okamura, S., Shimasaku, K., \& Brinkmann, J. 2004, ApJ, 128, 2677
Truong, N., Rasia, E., Mazzotta, P., et al. 2018, MNRAS, 474, 4089

Vogelsberger, M., Genel, S., Springel, V., et al. 2014, MNRAS, 444, 1518

Wang, L., Farrah, D., Oliver, S. J., et al. 2013, MNRAS, 431, 648

Weinmann, S. M., Lisker, T., Guo, Q., Meyer, H. T., \& Janz, J. 2011, MNRAS, 416, 1197

Welikala, N., Béthermin, M., Guery, D., et al. 2016, MNRAS, 455, 1629

Wetzel, A. R., Tinker, J. L., Conroy, C., \& van den Bosch, F. C. 2013, MNRAS, 432, 336

Wilman, D. J., Zibetti, S., \& Budavári, T. 2010, MNRAS, 406, 1701

Yates, R. M., Kauffmann, G., \& Guo, Q. 2012, MNRAS, 422, 215

Zhang, Y., Yang, X., Faltenbacher, A., et al. 2009, ApJ, 706, 747 